Lowbury, E. J. L. \& Hood, A. M. (1953). J. gen. Microbiol. 9, 524-535.

\title{
The Acquired Resistance of Staphylococcus aureus to Bacteriophage
}

\author{
By E. J. L. LOWBURY AND A. M. HOOD \\ Medical Research Council Industrial Injuries and Burns Research Unit, \\ Birmingham Accident Hospital
}

SUMMARY: To test the capacity of different staphylococei to acquire resistance to bacteriophage in vitro, twenty phages were grown on solid medium and in fluid medium with their propagating strains of Staphylococcus aureus, different phage types and patterns being represented.

Resistant secondary growth never appeared among staphylococci of the $3 \mathrm{~A}$ group and often appeared among members of the 6/47 group. Some members of the 29/52 group acquired resistance readily, and others never became resistant. Cross-resistance to other phages was commonly acquired. Secondary growth was shown to be resistant or sensitive to the phage, or to yield a mixture of resistant and sensitive colonies.

With the exception of propagating strain PS69, all staphylococci which became resistant to phage acquired lysogenicity for the sensitive parent strain. All but one of the staphylococci which had acquired resistance to a phage appeared to be capable of absorbing that phage. A substance which caused non-specific inhibition of phage lysis on agar medium was present in phage lysates of a staphylococcus that had shown sensitive secondary growth. These results are discussed with reference to phage typing and to the possibilities of therapy by phage.

During a trial of bacteriophage as a local prophylactic agent against Staphylococcus aureus in burns, swabs from the burns were found to contain an increasing proportion of non-typable staphylococci and of strains with a diminished sensitivity to a phage included in the prophylactic cream (Lowbury \& Hood, unpublished). Such a result might be expected if staphylococci on burns were acquiring resistance to the phage. Phage-resistant secondary growth of Staph. aureus in fluid cultures and on agar cultures has been described (Bruynoghe \& Maisin, 1921; Rountree, 1947; Williams Smith, 1948; Nicolle \& Conge, 1949). To assess the therapeutic potentialities of phage it would be desirable to know the capacity of a wide range of organisms to acquire resistance to it. In the experiments described below we examined the propagating strains of twenty different phages, (including representatives of the four groups; Williams \& Rippon, 1952), for the production of secondary growth in fluid and on solid media and the sensitivity of this secondary growth to phage. All strains were examined for acquired lysogenicity to the sensitive parent strains, and for cross-resistance to other phages. Resistant strains were also tested for ability to absorb the phage to which they had become resistant.

\section{MATERIALS AND METHODS}

Phages and strains of Staphylococcus aureus. Filtrates were obtained as described by Williams \& Rippon (1952) from the following phages: $3 \mathrm{~A}, 3 \mathrm{~B}, 3 \mathrm{C}$, $55,52,52 \mathrm{~A}, 42 \mathrm{E}, 47,70,54,7,75,29,31,6,53,42 \mathrm{D}, 69,44$ and $75 \mathrm{~B}$. The strains 
of Staph. aureus were those used for the routine propagation of the phages, and were designated by the same numbers with the prefix PS (propagating strain). The same propagating strain (PS 31/44) was used for phages 31 and 44. For the experiments described below, filtrates with a titre of not less than 1/1000 were used, the end-point chosen being semiconfluent lysis.

Culture media. Nutrient broth was used for fluid culture. The agar culture medium used for propagation and for typing was a modification of the one previously used by Hood (1953), and consisted of the following ingredients: Yeastrel, 3 g.; peptone (Allen \& Hanburys Eupepton no. 2), 5 g.; sodium chloride, 3 g.; sodium dihydrogen phosphate, 3 g.; shred agar, 12.5 g.; water, $1000 \mathrm{ml}$. This mixture was steamed, adjusted to $\mathrm{pH} \mathrm{8 \cdot 2-8 \cdot 4}$, filtered, readjusted to $\mathrm{pH} 7 \cdot 4-7 \cdot 6$, and autoclaved at $15 \mathrm{lb}$./sq.in. for $15 \mathrm{~min}$. Before use, $50 \mathrm{ml}$. horse serum were added to the melted agar; this enrichment was found to improve the propagation of most of the phages and the appearance of lysis.

Propagation of phage and cultivation of secondary growth in fluid culture medium. Two ml. of undiluted filtrate of each phage were mixed with an equal volume of an overnight broth culture of its propagating strain of Staph. aureus. After 3 days incubation at $37^{\circ}$ (and again after 6 days when phage-resistant growth was not found after 3 days) these tubes were examined for turbidity and subcultured to blood agar plates. Ten colonies from the growth appearing on each of these plates after incubation were subcultured in nutrient broth and typed with diluted and with undiluted phage filtrates by a modification of the method of Wilson \& Atkinson (see Hood, 1953).

Propagation of phage and cultivation of secondary growth on solid culture medium. On Yeastrel serum agar plates small areas were inoculated by wire loop with $18 \mathrm{hr}$. broth cultures of the nineteen strains of Staph. aureus. When these inocula had dried, loopfuls of undiluted phage filtrate were inoculated on their respective propagating strains of staphylococcus. The plates were incubated overnight at $37^{\circ}$ and examined for lysis and secondary growth. Whether secondary growth was present or not, a sterile loop was rubbed over the lysed area and inoculated into nutrient broth. After 4-5 hr. incubation at $37^{\circ}$, the broth tubes were subcultured to blood agar plates, which were incubated at $37^{\circ}$ for $24 \mathrm{hr}$. Ten colonies of Staph. aureus were then picked from each plate to nutrient broth, the growth in which was typed as described above.

Tests for lysogenicity of secondary growth. Ten colonies obtained by subculture from secondary growth of all strains, whether sensitive or resistant, were inoculated into nutrient broth. At least three subcultures were made in broth in order to eliminate transfer of phage from the original suspensions. Tubes of the last subculture were incubated at $37^{\circ}$ for $4-5 \mathrm{hr}$., and the contents were centrifuged for $10 \mathrm{~min}$. at 3000 r.p.m. The supernatant fluids were spotted on plates of Yeastrel serum agar, on which broth cultures of the sensitive parent staphylococci had been flooded and allowed to dry. These plates were incubated at $30^{\circ}$ overnight, then examined for lysis. Phages from resistant cultures shown to be lysogenic for the sensitive parent organisms were propagated in the usual way, and the lytic spectra were found (see Williams \& Rippon, 
1952). Similar tests of lysogenicity were made on ten colonies of subcultures from residual growth in broth cultures of staphylococci with phage.

Absorption of phage by staphylococci which acquired resistance. To determine whether staphylococci which have acquired resistance to a phage will absorb the same phage, 1 drop of undiluted phage filtrate was mixed with $5 \mathrm{ml}$. of a $24 \mathrm{hr}$. broth culture of Staph. aureus. Immediately $0.1 \mathrm{ml}$. of this mixture was transferred to $0.9 \mathrm{ml}$. broth and the diluted suspension was centrifuged. The phage particle concentration in the supernatant was estimated from plaque counts on plates of the propagating strain spotted with $0.02 \mathrm{ml}$. amounts of tenfold dilutions of the phage suspension. The approximate number of phage particles/ml. supernatant was also obtained after incubation of the mixture for 1 and $6 \mathrm{hr}$. at $37^{\circ}$. Phage particle counts were also made on control phage suspensions which were incubated for $6 \mathrm{hr}$. without staphylococci.

\section{RESULTS}

\section{Secondary growth on solid and in fluid media}

From Table 1 it can be seen that visible secondary growth appeared in the zone of lysis of all cultures on agar medium, with the exception of PS3A, PS3B, PS58 and PS42D; the secondary growth of PS31/44 and PS69 was poor. In fluid culture complete lysis was not found in any of the mixtures of staphylococci and phage. The organisms in this turbid medium might therefore consist either of secondary growth or of a mixture of residual organisms not attacked by the phage and secondary growth which occurred after the addition of phage.

\section{Phage sensitivity of secondary growth}

The results are summarized in Tables 1 and 2. In the experiments with agar medium, residual organisms from the zones of lysis of the four staphylococcal strains showing no visible secondary growth were detected on subculture in broth, and tested in the same manner as subcultures of secondary growth from other staphylococci.

Secondary or residual growth of PS 3 A, PSBB, PS3C, PS55, PS52, PS52A and PS42E from propagation either on solid or in fluid medium was never found to be resistant to the corresponding phages. On the other hand, secondary or residual growth of PS7 and PS75 from solid or fluid medium was always found to be resistant to phages 7 and 75 respectively. PS53 showed resistant secondary growth in broth, and no growth from subculture of zones of lysis on agar. The other staphylococci yielded a mixture of sensitive and resistant colonies in the subcultures from secondary or residual growth. The proportions of sensitive and resistant colonies obtained from solid and fluid media varied widely. PS 31/44 with phage 44 gave entirely different results by the two methods; subcultures from zones of lysis on agar yielded ten colonies resistant to phage 44, while forty colonies from secondary or residual growth of this organism in broth were sensitive to the phage. PS75B, in contrast, gave 10/10 sensitive colonies from secondary growth on agar and 9/10 resistant colonies from broth. 


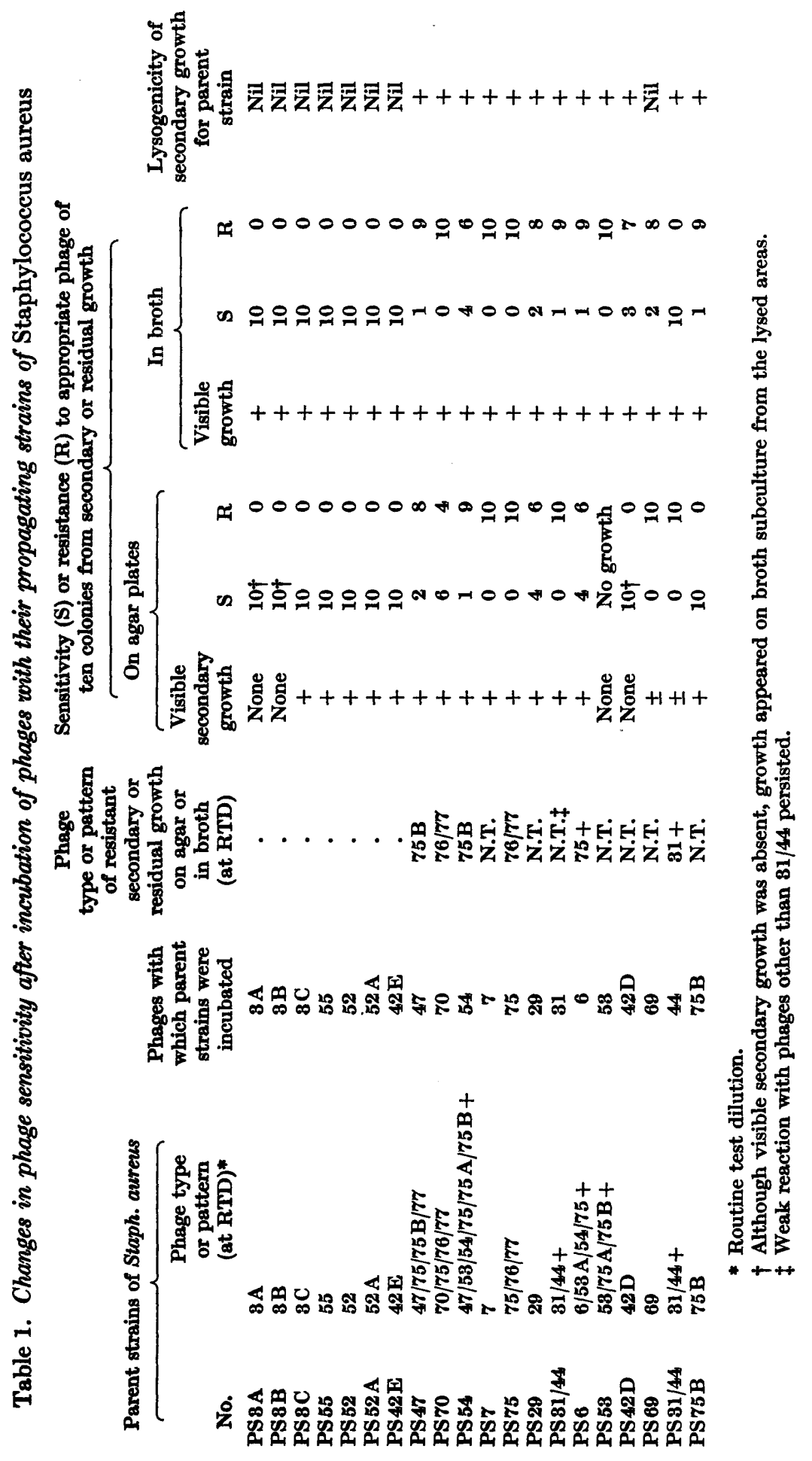




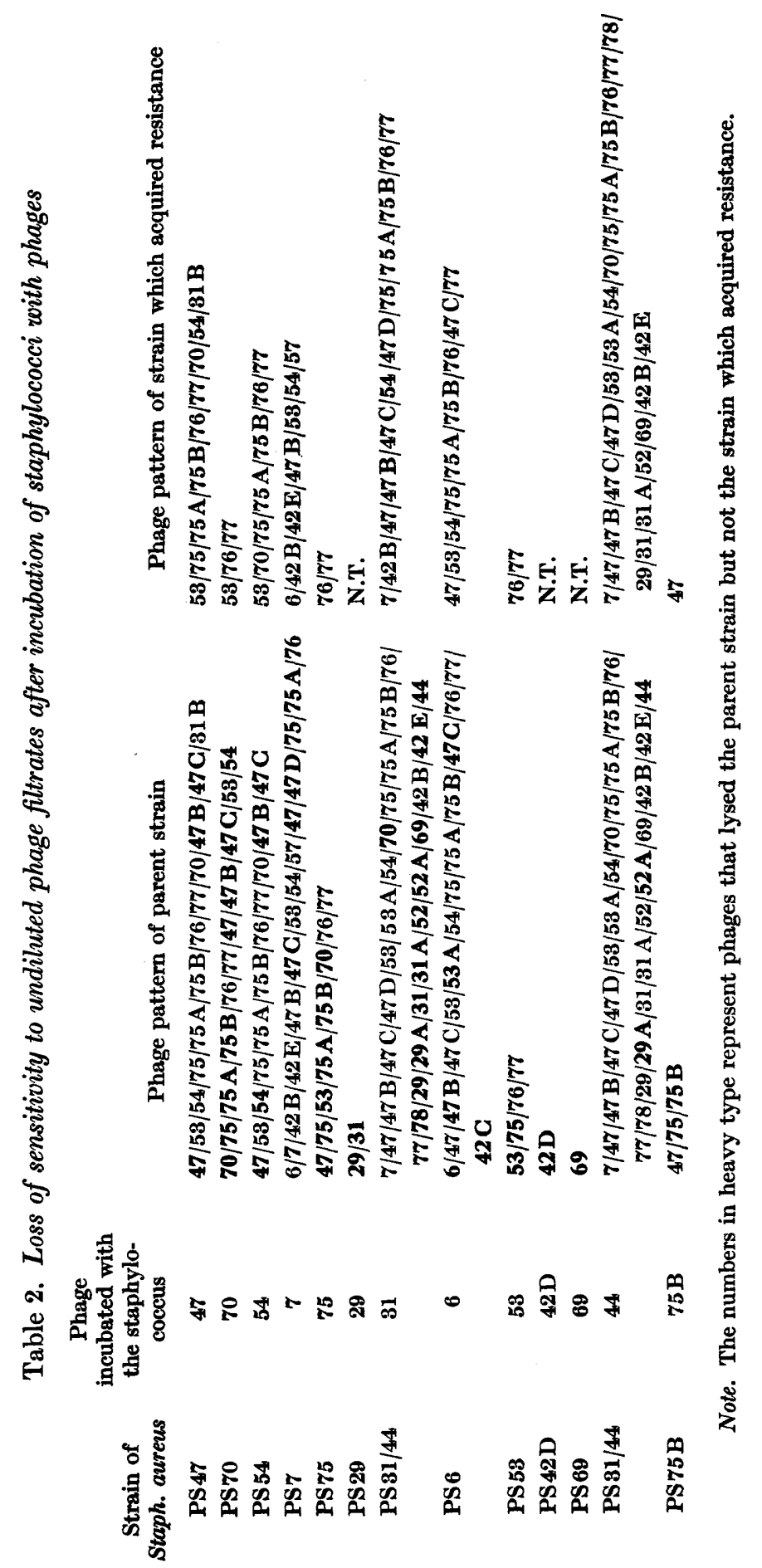




\section{Stability of acquired resistance}

After twenty serial subcultures, alternately from colonies on blood agar and from broth, PS 75 which had acquired resistance to phage showed no signs of a reversion to sensitivity. Acquired resistance and lysogenicity were also found to persist after at least six serial subcultures of the other strains described above.

\section{Cross-resistance of staphylococci to phages other than those propagated upon them}

The phage type of sensitive secondary growth, tested with filtrates at the routine test dilution (RTD) or undiluted, was the same as that of the parent strain. The phage type or pattern of resistant secondary growth, however, was shown to differ from that of the parent strain by the loss of sensitivity to the phage used in attempts to induce resistant secondary growth, and sometimes also to other phages (see Table 2). For example, Staph. aureus PS75 incubated with phage 75 lost its sensitivity to undiluted filtrates of phages $47,75,53,75 \mathrm{~A}, 75 \mathrm{~B}$ and 70 , retaining sensitivity to phages 76 and 77 only. Resistant secondary growth on agar and in broth showed the same changes in phage pattern. In no instance did a staphylococcus remain sensitive to the phage propagated upon it and acquire resistance to other phages. There was some tendency for resistance to be acquired towards closely related phages (e.g. 75 and $75 \mathrm{~A} ; 47,47 \mathrm{~B}$ and $47 \mathrm{C}$ ).

\section{Derivation of non-typable from typable staphylococci}

Resistant secondary growth from strains PS29, PS42D, PS69, PS7, PS 31/44, PS 53, and PS75B was non-typable by phages at the RTD; the first three of these were also non-typable by undiluted phages.

\section{Lysogenicity of secondary grozoth of Staphylococcus aureus}

With the exception of PS69, all the strains of Staph. aureus which had acquired resistance to the propagated phage were found to be lysogenic towards the parent strains. The lytic spectra of the phages propagated from the resistant organisms on the parent strains were identical with those of the phages against which the organism had been made resistant (e.g. the resistant secondary growth of PS75 carried a phage with lytic spectrum the same as that of phage 75). Sensitive secondary growth was not found to be lysogenic for the parent strain.

\section{Absorption of phages by strains which acquired resistance to them}

The number of phage particles remaining in suspension after $1 \mathrm{hr}$. and after $6 \mathrm{hr}$. incubation with the resistant staphylococci are shown in Table 3. There was considerable variation in the degree of absorption, ranging from complete absorption of phage 69 to an apparent failure of absorption of phage 70. The degree of absorption was not related to the concentration of the 


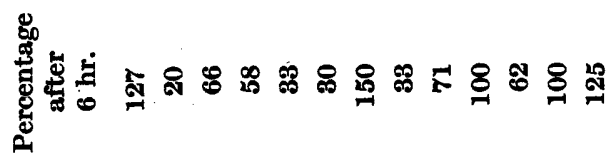

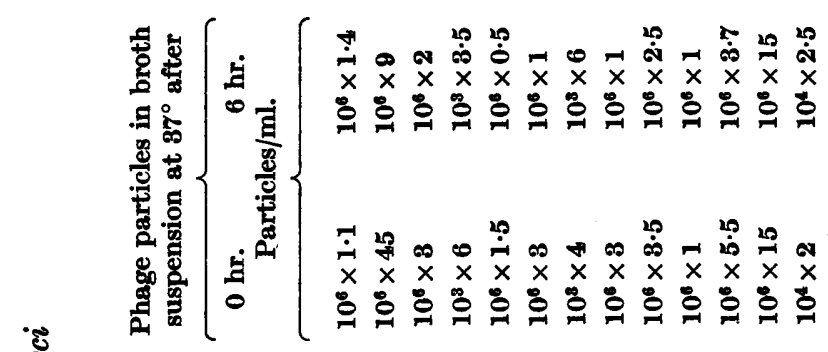

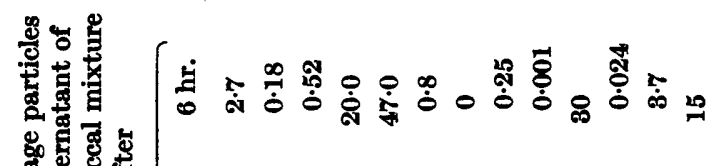

施

更

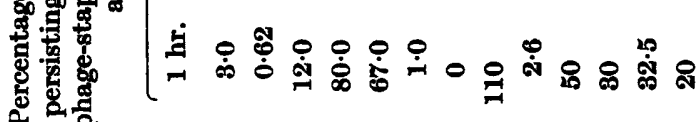

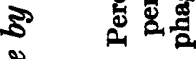

हूँ

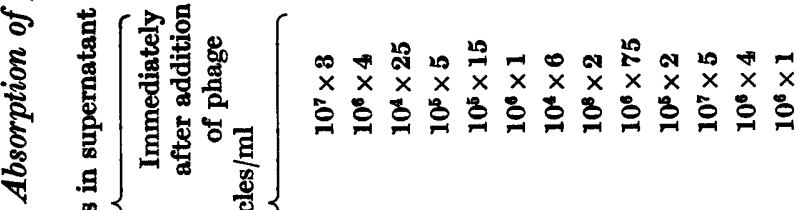

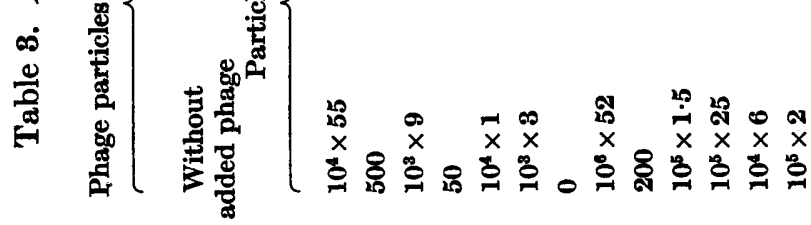

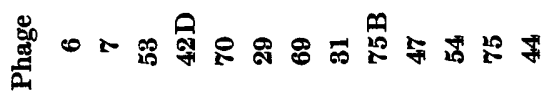

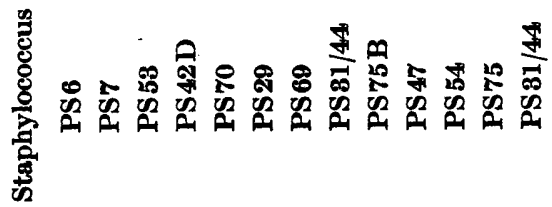


phage in the lysogenic bacterial suspension before the addition of phage. Nevertheless, the only staphylococcus which was not lysogenic for the sensitive parent strain (PS 69) effected the most complete absorption of phage.

\section{The emergence of secondary phage-sensitive organisms}

Phage inhibitors in extracts of staphylococci have been described by Rakieten, Rakieten \& Doff (1936) and by Freeman (1937). It seemed to us likely that the appearance of secondary phage-sensitive organisms in our cultures might be due to such inhibitors appearing in the cultures. To test this hypothesis we made the following experiments. Phages 75B, 69 and 44 and their propagating strains of Staph. aureus were chosen because they

\section{Table 4. The inhibition of phage lysis on agar by application of additional phage filtrate}

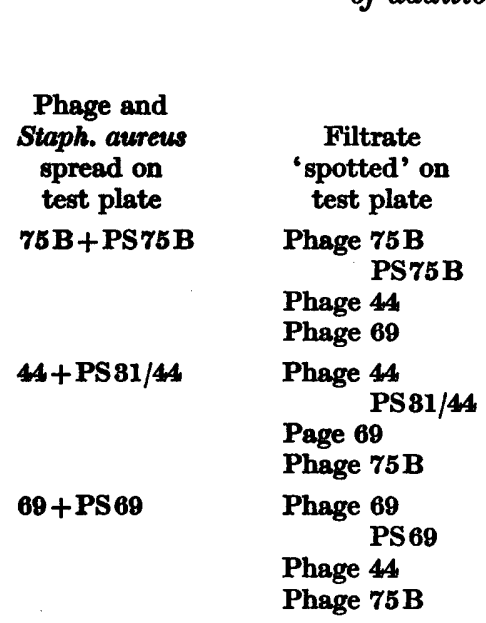

Presence of zone of heavier growth
where filtrate was 'spotted', and its sensitivity to appropriate phage

$\begin{array}{cl}\text { From agar } & \text { From broth } \\ \text { propagation } & \text { propagation } \\ +++ \text { (sensitive) } & + \text { (sensitive) } \\ \mathbf{0} & 0 \\ \mathbf{0} & 0 \\ 0 & 0 \\ 0 & + \text { (sensitive) } \\ \mathbf{0} & 0 \\ \mathbf{0}++ \text { (sensitive) } & 0 \\ + \text { (resistant) } & 0 \\ \mathbf{0} & 0 \\ 0 & 0 \\ ++ \text { (sensitive) } & \pm \text { (sensitive) }\end{array}$

presented three different patterns in the phage sensitivity of secondary or residual growth (see Table 1). Agar plate propagations of the three phages were set up in the usual way. After $6 \mathrm{hr}$. incubation at $37^{\circ}$, the plates were placed in the refrigerator freezing unit and removed after $18 \mathrm{hr}$. to thaw at room temperature. The fluid pipetted from the thawed plates was centrifuged and filtered through a Seitz E.K. pad. Broth propagations were carried out by incubating equal volumes of undiluted phage suspension and broth culture of the propagating strain at $87^{\circ}$. After 3 days incubation the suspensions were centrifuged and filtered. A part of each filtrate was heated at $52^{\circ}$ for $1 \mathrm{hr}$. Control filtrates from staphylococcal cultures without added phage were also obtained. These filtrates were tested for the presence of inhibitors of phage lysis by 'spotting' them on plates newly inoculated as for phage propagation but with higher concentrations (usually $\times 10$ ) of phage. All three phages were spotted on each propagation plate; the plates were then incubated overnight at $87^{\circ}$ and examined. The principal findings are shown in Table 4 and in Pl. 1, figs. 1-8. On each plate there was diffuse lysis with a fine layer of 
secondary growth of the propagating strains, PS75B, showing rather more secondary growth than PS69 and PS31/44. In addition, the following appearances were observed:

At the site where filtrates from agar propagation of phage were 'spotted'. On all the plates disks of heavy growth appeared where phage $75 \mathrm{~B}$ filtrate was spotted. A slight accentuation of growth occurred where phage 69 filtrate was spotted on the plate with phage 69 and PS69 $(69+/$ PS69). No similar increase of growth occurred where this phage was spotted on plates of 44 + PS31/44 and 75B +/PS75B. Phage 44 filtrate caused increased lysis where it was spotted on plate $44+\mathrm{PSB1} / 44$, but in other respects resembled phage 69 filtrate. Ten colonies subcultured from the disks of heavy growth in the areas spotted with phage 75 B filtrate were found to be sensitive to their corresponding phages. The slightly accentuated growth of PS69 in the area spotted with phage 69 was resistant to this phage. The diffuse secondary growth on the propagation plate of phage 75 $\mathrm{B}$ was sensitive, but that on the propagation plates for phages 44 and 69 was resistant to the propagated phage.

At the site where filtrates from broth were 'spotted'. Some increase in staphylococcal growth was found on the propagation plate for phage 44 where filtrate from the broth propagation of phage 44 had been 'spotted'. Subcultures from this growth were sensitive to phage 44. A similar increase in growth occurred where phage $75 \mathrm{~B}$ broth propagation was 'spotted' on a propagation plate of 75B. Neither of these filtrates caused increased growth on plates spread with the other staphylococci and phages. No increase of growth appeared on any plate at the site of application of phage 69 broth filtrate.

Action of the phage inhibitor on twelve different phages. Filtrate from agar plate propagation of phage $75 \mathrm{~B}$ was applied to areas on an agar plate previously inoculated with propagating strains and phages (at RTD) 6, 7, 47, $47 \mathrm{~A}, 47 \mathrm{C}, 31 \mathrm{~A}, 31 \mathrm{~B}, 53,53 \mathrm{~A}, 71,76$ and $75 \mathrm{~B}$. A heavy growth of staphylococci appeared in all these areas; by contrast a control plate with no added phage 75 B showed confluent lysis with little or no growth of staphylococci. These results show that the phage inhibitor is not specific for the staphylococcus and phage from which it was obtained.

Local application of other filtrates. Lysis was not inhibited by filtrates from the control agar or broth cultures of staphylococci without phage or from heated filtrates from agar or broth propagation of phage. Stock phages 75B, 69 and 44 used for routine typing of staphylococci gave the same results as the special agar plate propagations used in the experiment.

Interpretation of results. Lysis by several different phages was inhibited in the presence of filtrates from agar propagation of phage $75 \mathrm{~B}$, and smaller amounts of inhibitor could also be detected in a filtrate of a broth propagation of this phage. No such inhibitor was present in the filtrates from agar propagations of phages 44 and 69. A small amount of the inhibitor was, however, present in a filtrate from broth propagation of phage 44. The titre of phage in this filtrate and that from broth propagation of phage 75 B was much lower than that obtained from the agar plate propagation. The increased growth of 
PS69 at the site of application of phage 69 filtrate was resistant to the phage, unlike that which occurred where $75 \mathrm{~B}$ was applied; it appeared therefore to be caused not by an inhibitor of phage lysis but by some other mechanism.

\section{DISCUSSION}

In our experiments different phage types of Staph. aureus differed widely in their capacity to acquire resistance to the phages propagated on them. In particular, no evidence of acquired resistance was shown by any of the staphylococci in the phage group $3 \mathrm{~A}$, whereas staphylococci of the $6 / 47$ phage group acquired resistance very readily. The behaviour of the groups in this respect seems analogous to their behaviour in relation to penicillin and to aureomycin. Penicillin-resistant Staph. aureus strains isolated from patients in hospital are most commonly found to belong to the 6/47 group (Barber \& Whitehead, 1949; Lowbury, Topley \& Hood, 1952). In this M.R.C. Unit, during a period when both penicillin and aureomycin were used routinely, the great majority of staphylococci showed certain patterns of the 6/47 group and were resistant to penicillin and many also to aureomycin. Most of the small number of staphylococci in the $3 \mathrm{~A}$ group which were isolated from burns during this time were sensitive to penicillin and to aureomycin. Barber \& Whitehead (1949) suggest the possibility that strains of the 6/47 group are genetically less stable than those of the other phage groups. This suggestion gains support from our finding that staphylococci in the 6/47 group have a greater capacity than other staphylococci to acquire resistance to phage:

D'Herelle (1922) recognized both lysogenic and non-lysogenic strains among organisms which had acquired resistance to phage. In our study, all but one of the staphylococci (PS69) were found to have become lysogenic for the parent strain when they became resistant to the phage. Absorption of phage was shown in varying measure by all but one of the staphylococci which had acquired resistance; in this respect their behaviour is typical of staphylococci, which are known to absorb phages that do not lyse them (Burnet \& Lush, 1935; Rountree, 1947; Hood, 1953). Our result, however, does not corroborate the assumption of Delbrück (1946) that 'resistance is primarily caused by a loss of the capacity for adsorbing a specific virus'.

The secondary growth which appears in the zone of lysis may be either resistant or sensitive to the phage, or consist of a mixture of sensitive and resistant organisms. The appearance of secondary sensitive growth could be explained if inhibitors of phage lysis were liberated in the medium on which staphylococci were lysed by phage. Rakieten et al.(1936) and Freeman (1937) described phage inhibitors in extracts of staphylococci. Our experiments showed the presence of such an agent in filtrates from the agar propagation of phage 75B on its propagating strain of Staph. aureus, an organism which had shown secondary sensitive growth in zones of lysis. Similar filtrates of phages 44 and 69, however, were not found to contain such an inhibitor of phage lysis; this observation was consistent with the absence of sensitive secondary growth in zones of lysis produced by phages 44 and 69 on their 
propagating strains of Staph. aureus. Filtrate from the plate propagation of phage 75B was found to inhibit phage lysis of a number of different staphylococci, including that of PS 69 by phage 69 and of PS31/44 by phage 44. The inhibitor is therefore not specific in its action. It differs from the phage inactivator described by Rakieten et al. in being thermolabile. Some increased growth of PS69 occurred at the site of application of a filtrate of phage 69; this growth, unlike that stimulated by applying a filtrate of phage 75B, was resistant to phage 69 , and must be considered to have appeared through some other mechanism, e.g. the quicker emergence of resistant organisms where phage particles are more abundant. Discrepancies between the phage sensitivity of secondary or residual growth on agar and in broth are consistent with the results of these experiments, e.g. evidence of an inhibitor of phage lysis could be found in filtrates from broth but not from agar propagation of phage 44; filtrates from agar propagation of phage $75 \mathrm{~B}$, on the other hand, showed evidence of a greater concentration of inhibitor than could be found in filtrate from broth propagation. Further study is being made on this phenomenon.

Our results, like those of Williams Smith (1948), show that considerable difference in the phage pattern of staphylococci may occur as the result of acquired resistance. Staphylococci which are lysed by a 'pattern' of phages may become resistant to several of them after growing in the presence of one phage; they may also become non-typable. The possibility that such changes may occur in the environment (Williams Smith, 1948) shows the severe limitation of phage pattern as a descriptive label for staphylococci.

Successful phage therapy might be expected in infection by staphylococci of the phage group $\mathbf{B A}$, or in other staphylococci which do not readily acquire resistance to the phage. But since these are probably the organisms that also more readily retain their sensitivity to the antibiotics, phages are unlikely to contribute much to the therapy or prophylaxis of staphylococcal infections.

\section{REFERENCES}

Barber, M. \& Whitehead, J. E. M. (1949). Bacteriophage types in penicillinresistant staphylococcal infection. Brit. med. J. ii, 565 .

Bruynoghe, R. \& Maxsin, J. (1921). Le principe bactériophage du staphylococque. C.R. Soc. Biol., Paris, 85, 1118.

Burnet, F. M. \& Lush, D. (1935). The staphylococcal bacteriophages. J. Path Bact. 40, 455.

DeLbrück, M. (1946). Bacterial viruses or bacteriophages. Biol. Rev. 21, 30.

Fraeman, M. (1937). The phage inactivating agent in extracts of Staphylococcus aureus. Aust. J. exp. Biol. med. Sci. 15, 221.

d'Hereme, F. (1922). The Bacteriophage: its Role in Immunity. (Quoted in Hadley, P. (1928), J. infect. Dis. 42, 263.)

Hoon, A. M. (1953). Phage typing of Staphylococcus aureus. J. Hyg., Camb. 51, 1.

Lowbury, E. J. L., Topley, E. \& Hood, A. M. (1952). Chemotherapy for Staphylococcus aureus in burns. Lancet, $i, 103 \theta$.

Nrcolle, P. \& Conge, G. (1949). Le théorie des mutants spontanés: est elle applicable a tous les cas de cultures secondaires après bactériophagie? Ann. Inst. Pasteur, 76, 381.

Rakieten, M. L., Rakieten, T. L. \& Doff, S. (1936). Absorption of staphylococcal phages. J. Bact. 32, 505. 
Journal of General Microbiology, Vol. 9, No. 3
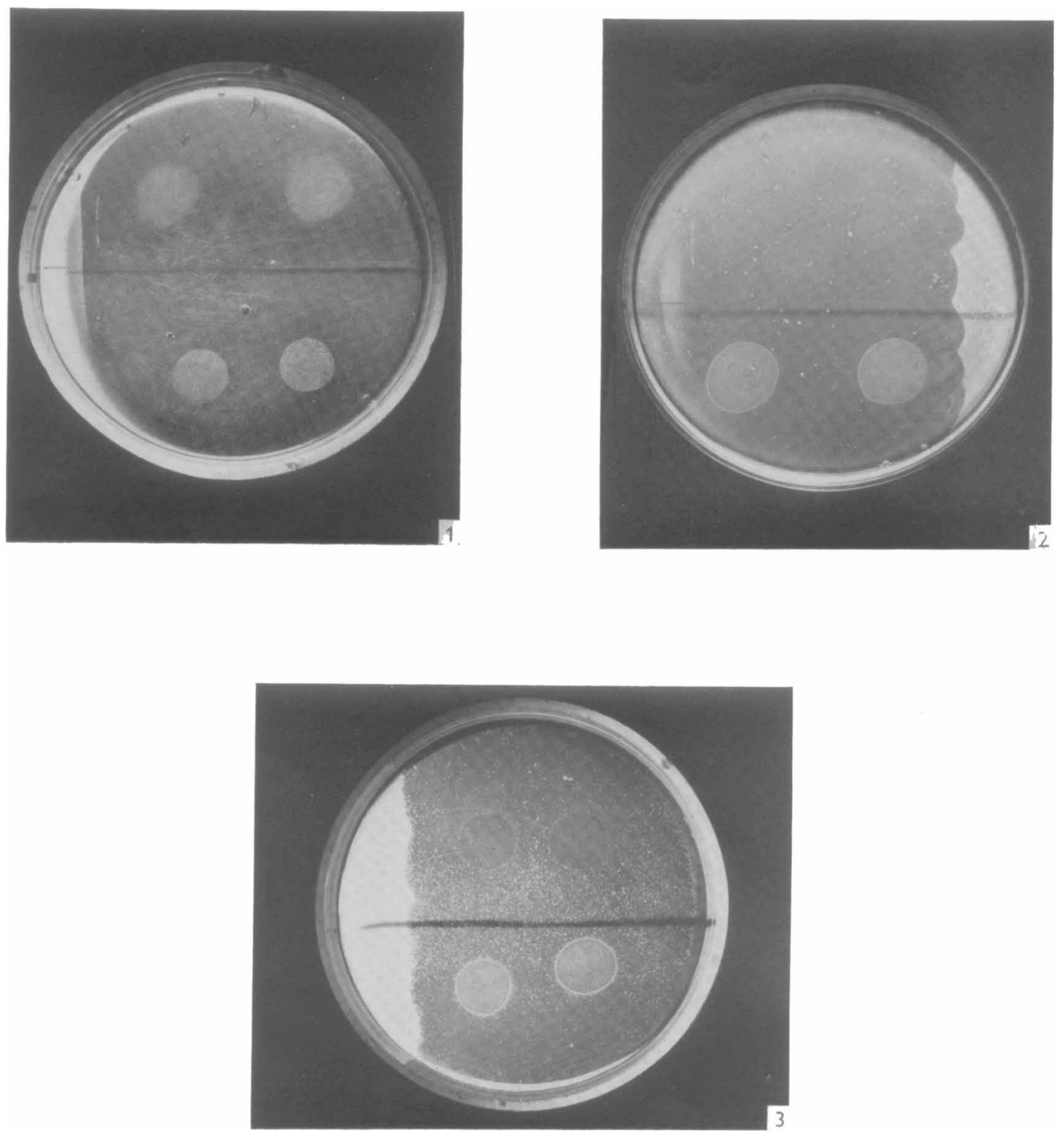

E. J. L. Lowbury and A. M. Houd-Acquired resis'tance to bac'meliopiage. Phate 1 
Rountrak, P. (1947). Bacteriophage absorption by staphylococci. Aust. J. exp. Biol. med. Sci. 25, 203.

Wrumams, R. E. O. \& Rippon, J. (1952). Bacteriophage typing of Staphylococcus aureus. J. Hyg., Camb. 50, 320.

Wrimams Smrth, H. (1948). Investigations on the typing of staphylococci by means of bacteriophage. J. Hyg., Camb. 46, 74, 82.

\section{EXPLANATION OF PLATE}

Fig. 1. Propagation plate of phage 69 on PS69. The two zones of heavier growth in the upper half of the plate are at the site of application of phage 69 filtrate, and those in the lower half are at the sites of application of phage 75B filtrate, both filtrates having been applied before incubation of the plate.

Fig. 2. Propagation plate of phage 75B on PS75B. In the lower half of the plate are two zones of heavier growth where phage 75B fltrate was 'spotted' before incubation of the plate. No zones of heavier growth appeared in the upper half of the plate, where phage 69 filtrate was applied before the plate was incubated.

Fig. 8. Propagation plate of phage 44 on PS31/44. As in figs. 1 and 2, zones of heavier growth appeared in the lower half of the plate where phage 75B filtrate was applied before incubation. In the upper half of the plate are two areas of increased lysis at the site of application of phage $\mathbf{4}$ f filtrate before incubation.

(Received 22 June 1953) 